OPEN ACCESS

Edited by:

Ester Boix,

Autonomous University of

Barcelona, Spain

Reviewed by:

John David Spencer,

Nationwide Children's Hospital,

United States

David Hains,

Riley Hospital for Children,

United States

Bruno R-s,

Unidad de Investigación Biomédica de

Zacatecas (IMSS), Mexico

*Correspondence: Jürgen Harder

jharder@dermatology.uni-kiel.de

tThese authors share first authorship

Specialty section:

This article was submitted to Microbial Immunology, a section of the journal

Frontiers in Immunology

Received: 09 August 2019 Accepted: 15 October 2019 Published: 05 November 2019

Citation:

Rademacher F, Dreyer S, Kopfnagel $V$, Gläser R, Werfel T and Harder J

(2019) The Antimicrobial and

Immunomodulatory Function of RNase

7 in Skin. Front. Immunol. 10:2553.

doi: 10.3389/fimmu.2019.02553

\section{The Antimicrobial and Immunomodulatory Function of RNase 7 in Skin}

\author{
Franziska Rademacher ${ }^{1 \dagger}$, Sylvia Dreyer ${ }^{2 \dagger}$, Verena Kopfnagel ${ }^{2,3}$, Regine Gläser ${ }^{1}$, \\ Thomas Werfel ${ }^{2,4}$ and Jürgen Harder ${ }^{1 *}$
}

\begin{abstract}
${ }^{1}$ Department of Dermatology, University of Kiel, Kiel, Germany, ${ }^{2}$ Division of Immunodermatology and Allergy Research, Department of Dermatology and Allergy, Hannover Medical School, Hanover, Germany, ${ }^{3}$ Hannover Unified Biobank, Hannover Medical School, Hanover, Germany, ${ }^{4}$ Cluster of Excellence RESIST (EXC 2155), Hannover Medical School, Hanover, Germany
\end{abstract}

The human ribonuclease RNase 7 has been originally isolated from human skin and is a member of the human RNase A superfamily. RNase 7 is constantly released by keratinocytes and accumulates on the skin surface. The expression of RNase 7 in keratinocytes can be induced by diverse stimuli such as cytokines, growth factors, and microbial factors. RNase 7 exhibits a potent broad spectrum of antimicrobial activity against various microorganisms and contributes to control bacterial growth on the skin surface. The ribonuclease and antimicrobial activity of RNase 7 can be blocked by the endogenous ribonuclease inhibitor. There is also increasing evidence that RNase 7 exerts immunomodulatory activities and may participate in antiviral defense. In this review, we discuss how these characteristics of RNase 7 contribute to innate cutaneous defense and highlight its role in skin infection and inflammation. We also speculate how a potential dysregulation of RNase 7 promotes inflammatory skin diseases and if RNase 7 may have therapeutic potential.

Keywords: RNase 7, antimicrobial ribonucleases, RNA, innate immunity, cutaneous defense, skin infection, skin inflammation

\section{INTRODUCTION}

Human skin is permanently in contact with microorganisms. Among these microorganisms are many beneficial members of the microbiota. In addition, the skin has to deal with potential pathogenic microorganisms causing partially severe skin infections. There is increasing evidence that antimicrobial peptides and proteins (AMP) control the growth of microorganisms on all epithelial tissues including skin. AMP may play an important role to shape and control the preferred microbiota as well as to protect the skin from pathogenic microorganisms. Human skin is able to produce different types of AMP that exhibit individual activity profiles. One major AMP expressed by keratinocytes and released on the skin surface is the ribonuclease RNase 7. RNase 7 belongs to the human RNase A superfamily which consists of 13 genes located on chromosome 14 and contains eight different members with RNase activities (RNase 1-8) (1). The RNase A superfamily is named after its founding member, bovine pancreatic ribonuclease (RNase A). The members of this family contain an RNase catalytic domain that is composed of one lysine and two histidine residues. The protein structure is characterized by eight cysteine residues which are connected via four disulfide bridges (1). 
A huge repertoire of diverse physiological functions of these RNases has been reported during the last decades. Examples are angiogenic, neurotoxic, and diverse immunomodulatory activities (2). Several members of the human RNase A superfamily are also characterized by antimicrobial activities and there is increasing evidence that they participate in innate immunity. In particular, RNase 2 (also termed eosinophil-derived neurotoxin, EDN) and RNase 3 (also termed eosinophil cationic protein, ECP) exhibit diverse antiviral and antibacterial activities as well as immunomodulatory activities suggesting an important role in innate host defense $(3,4)$. Moreover, antimicrobial activities have also been reported for RNase 5 (also named angiogenin due to its angiogenic potency) (4-6), RNase 6 (7), RNase $7(8,9)$, and RNase $8(10)$. RNase 5 and RNase 7 are both expressed by keratinocytes and thus may contribute to cutaneous innate defense $(6,11)$. Indeed, there is increasing evidence that RNase 7 may play an important role to protect skin from infection (8). This review aims to summarize the current knowledge about the physiological activities of RNase 7 and to highlight its role in cutaneous host defense.

\section{EXPRESSION AND INDUCTION OF RNase 7 IN SKIN}

\section{Site of RNase 7 Expression}

The RNase 7 protein has been originally isolated from stratum corneum extracts during an attempt to identify and characterize the antimicrobial factors produced by human healthy skin (12). As the name implies, it is the seventh member of the human RNase A superfamily and exhibits ribonuclease activity. It has a molecular mass of $14.5 \mathrm{kDa}$ and is highly cationic due to the presence of many arginine and lysine residues (12). Isolation of the protein from human skin and subsequent cloning of the corresponding cDNA enabled the identification of RNase 7 gene expression in human skin-derived keratinocytes (12). Of note, RNase 7 is not only expressed in human skin. Gene expression analyses revealed expression in various tissues such as heart, liver, kidney, pharynx, and tonsil $(12,13)$. In addition, the group of Spencer et al. identified a major role of RNase 7 in kidney and bladder host defense $(14,15)$.

Immunostaining of skin biopsies revealed RNase 7 expression in the keratinocytes throughout all epidermal layers with an increased staining in the more differentiated uppermost layers and an accumulation in the stratum corneum (12, 16). These data are in concordance with expression analyses of cultured keratinocytes demonstrating a higher RNase 7 gene expression in differentiated cultures as compared with proliferating keratinocytes (6). RNase 7 immunoreactivity was also present in sebaceous glands and hair follicles $(12,17)$. Analysis of skin rinsing fluids revealed the presence of RNase 7 throughout various body areas confirming the constitutive expression and release of RNase 7 by keratinocytes $(16,18)$. Interestingly, RNase 7 is also expressed in fetal skin suggesting that RNase 7 may already control cutaneous microbial growth of the fetus and newborn (19). In addition, RNase 7 may have other, as yet unknown functions in fetal skin such as a regulatory role in development. However, this hypothesis remains to be proven.

\section{Induction of RNase 7 Expression}

In addition to the constitutive expression, RNase 7 expression in keratinocytes can be induced by various stimuli and different signal transduction pathways (summarized in Figure 1). Proinflammatory cytokines such as IL-17A, interferon-gamma and IL- 1 are able to induce the expression of RNase 7 in keratinocytes $(12,17,20,21)$. Especially the synergistic action of proinflammatory cytokines such as IL-17A and interferongamma markedly induced the expression of RNase 7 (20). The synergistic induction of RNase 7 by IL-17A/interferongamma was mediated by the signal transducer and activator of transcription 3 (STAT3) (20). Mohammed et al. reported that the induction of RNase 7 by IL-1beta in corneal epithelial cells was mediated by TGFbeta-activated kinase-1 (TAK-1). This in turn led to activation of the mitogen-activated protein kinase (MAPK) pathway resulting in activation of the transcription factors $\mathrm{c}$ Jun and activating transcription factor 2 (ATF2). In contrast, the NF-kappaB pathway had no major influence on the IL-1betamediated induction of RNase 7 in corneal epithelial cells (21). Induction of RNase 7 by pro-inflammatory cytokines was also detected in the keratinocytes cell line HaCaT (22). Overall, the induction of RNase 7 by proinflammatory cytokines suggests that the local environment of inflammatory skin diseases like psoriasis trigger the increased expression of RNase $7(23,24)$.

Since RNase 7 exhibits potent antimicrobial activity (see below), it would be of biological significance that keratinocytes respond with an increased RNase 7 expression to the presence of microorganisms, in particular potential pathogens. Indeed, the expression of RNase 7 in keratinocytes can be induced by Staphylococcus (S.) aureus (25-28), Pseudomonas (P.) aeruginosa (29), Enterococcus (E.) faecium (16), and the dermatophyte Trichophyton (T.) rubrum (30). Of note, also skin commensals such as Staphylococcus (S.) epidermidis and Corynebacterium (C.) amycolatum induce RNase 7 expression in keratinocytes indicating that the presence of commensals leads to an increased RNase 7 production thereby strengthening cutaneous defense $(22,27,31) . S$. epidermidis also enhanced the S. aureus-induced expression of RNase 7 in keratinocytes suggesting that the presence of commensals amplifies the defense response of human keratinocytes toward pathogens such as S. aureus (27). Interestingly, the induction of RNase 7 by the skin commensals S. epidermidis (27) and C. amycolatum (31), and by P. aeruginosa (29) and T. rubrum (30) depends on the involvement of the epidermal growth factor receptor (EGFR). This suggests that the EGFR plays an important role in cutaneous defense by its crucial role to mediate the expression of AMP such as RNase 7 and may offer an explanation for the increased susceptibility for skin infections of cancer patients receiving anti-EGFR therapy (32). Wanke et al. reported that-in addition to the EGFR-also Toll-like receptor-2 (TLR-2) and the transcription factor NF-kappaB are involved in the induction of RNase 7 in keratinocytes simulated with culture supernatants of $S$. epidermidis. This is in contrast to $S$. aureus which has been 


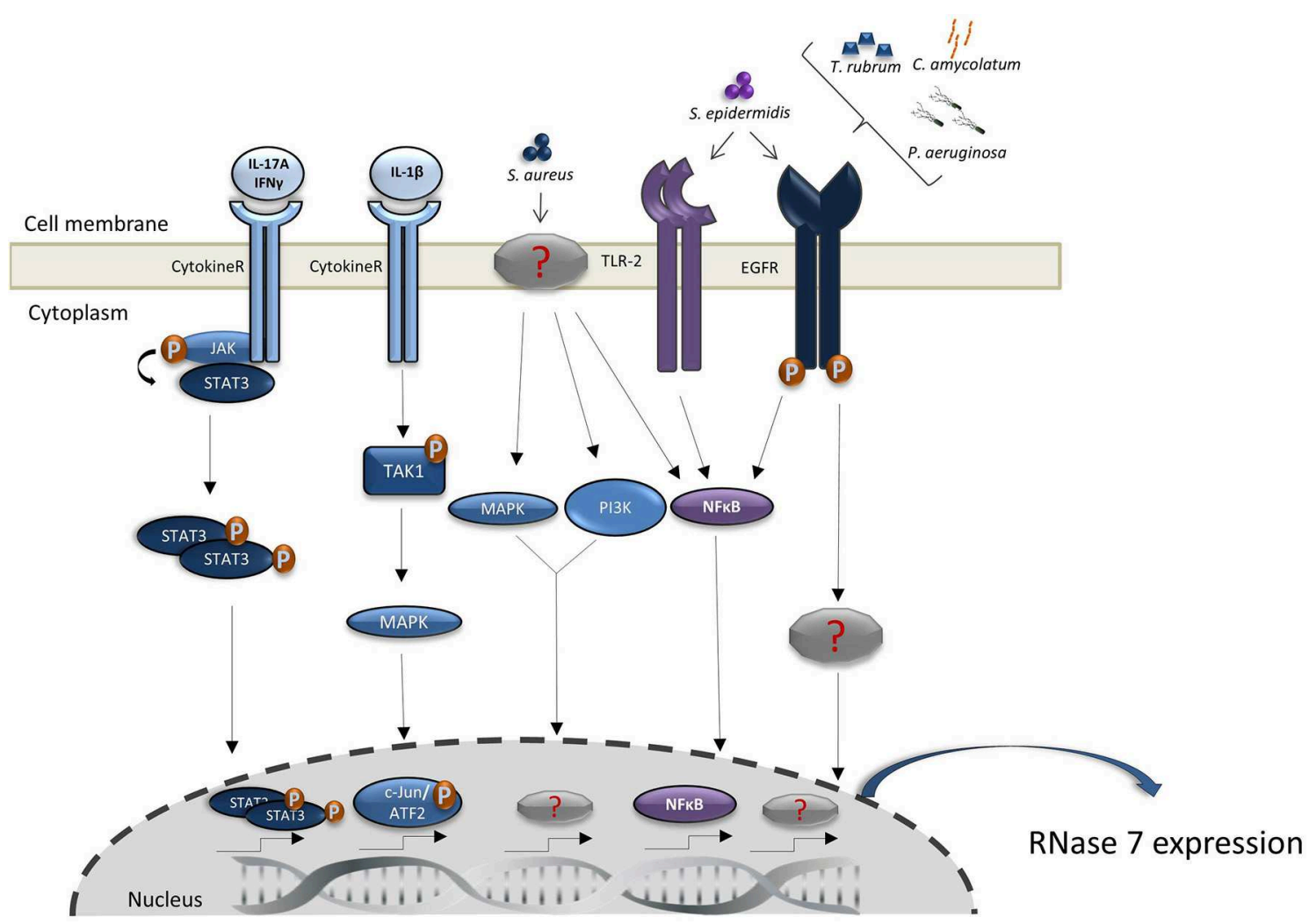

FIGURE 1 | Regulation of RNase 7 induction. Shown are the proposed signal transduction pathways implicated in the induction of RNase 7 by microorganisms and cytokines. Detailed explanations are given in the main text.

reported to activate the MAPK and phosphatidylinositol 3kinase/AKT signaling pathways to induce RNase 7 expression (27). Thus, it seems that commensal and pathogenic bacteria activate different signal transduction pathways to induce RNase 7 expression in keratinocytes. RNase 7 expression in keratinocytes can also be induced by Borrelia burgdorferi, a bacterium that causes Lyme borreliosis and is transmitted by Ixodes ticks. Interestingly, tick saliva proteins have been shown to inhibit the Borrelia burgdorferi-mediated induction of RNase 7 and of other antimicrobial peptides and chemokines. This suggests that components of the tick saliva inhibit cutaneous innate defense reactions against Borrelia burgdorferi and thereby help the bacterium to evade local host defense and to disseminate into the body (33).

The role of cutaneous RNase 7 expression in the context of viral infection is less explored. It has been reported that keratinocytes infected with dengue virus showed an increased RNase 7 expression. If this is a direct induction or indirectly mediated by the release of inflammatory mediators is not clear. In addition, if RNase 7 influences the infectivity by dengue virus has not been reported (34). UV-B radiation also induces the expression of RNase 7 and other AMP in keratinocytes. This induction may contribute to the UV-mediated strengthening of the innate immune response (35). Niacinamide has also been identified as an inducer of the expression of RNase 7 and other antimicrobial peptides in human keratinocytes. Niacinamide is a well-known cosmetic ingredient and its antimicrobial peptidesinducing capacity may account for its observed beneficial effects on the skin barrier (36).

\section{ANTIMICROBIAL AND RIBONUCLEASE ACTIVITY}

\section{Antimicrobial Spectrum of RNase 7}

A key feature of RNase 7 is its high antimicrobial activity against a wide spectrum of microorganisms. In vitro studies showed that RNase 7 is highly antimicrobial effective in low micromolar concentrations against Gram-positive and Gramnegative bacteria like $S$. aureus, $P$. aeruginosa, C. amycolatum, E. faecium, Mycobacterium vaccae, the yeast Candida (C.) albicans and Pichia pastoris and the dermatophyte T. rubrum (12, 16, 30, 37-41). The known microorganisms susceptible to RNase 7 are listed in Table $1(6,7,12,14-16,25,29-31,37-50)$. The functional relevance of the antimicrobial activity of RNase 7 and its contribution to the antimicrobial capacity of stratum corneum was demonstrated by the use of antibodies that neutralized the antimicrobial activity of RNase 7. Using such antibodies, we could show that inactivation of RNase 7 in human stratum corneum extracts led to an increased outgrowth of $P$. aeruginosa, S. aureus, 
TABLE 1 | Overview of the microorganism susceptible to RNase 7.

\begin{tabular}{lc}
\hline Microorganism & References \\
\hline GRAM-POSITIVE BACTERIA & $(12,25,37,38,42-46)$ \\
Staphylococcus aureus & $(7,47)$ \\
Staphylococcus saprophyticus & $(6,12,16,44)$ \\
Enterococcus faecium & $(6,7,48)$ \\
Enterococcus faecalis & $(12)$ \\
Propionibacterium acnes & $(44)$ \\
Micrococcus luteus & $(31)$ \\
Corynebacterium amycolatum & $(31)$ \\
Corynebacterium xerosis & $(12,14,29,37,44,48,49)$ \\
GRAM-NEGATIVE BACTERIA & $(7,12,14,15,37,44,46-48)$ \\
Pseudomonas aeruginosa & $(13,22)$ \\
Escherichia coli & $(44)$ \\
Proteus mirabilis & $(14)$ \\
Acinetobacter baumannii & $(39)$ \\
Klebsiella pneumoniae & $(50)$ \\
MYCOBACTERIA & $(40)$ \\
Mycobacterium tuberculosis & $(39)$ \\
Mycobacterium vaccae & $(37)$ \\
YEAST & \\
Candida albicans & $(39,39)$ \\
Pichia pastoris & \\
DERMATOPHYTES & \\
Trichophyton rubrum & \\
Trichophyton mentagrophytes & \\
Microsporum canis & \\
Epidermophyton floccosum & \\
\hline & \\
\hline
\end{tabular}

C. amycolatum, and E. faecium. These results demonstrated that RNase 7 is part of the growth control mechanism in the stratum corneum $(16,25,29,31)$.

The Gram-positive bacterium S. aureus is a principal skin pathogen causing many infectious cutaneous diseases. Our work with ex vivo skin explants infected with $S$. aureus showed an increased release of RNase 7 expression. Inhibition of the antimicrobial activity of RNase 7 by specific antibodies resulted in considerable outgrowth of $S$. aureus on the skin surface. These results accentuate the functional relevance of RNase 7 in cutaneous defense against hazardous skin pathogens (25). This goes in line with a study from Zanger et al. who analyzed the RNase 7 expression levels of unaffected skin from healthy control persons and subjects with a $S$. aureus skin infection after a journey to a tropic or subtropic destination. They detected a 64\% higher RNase 7 expression level in skin of the control group than in the unaffected skin of the infected persons. These data suggest that high RNase 7 baseline levels in healthy skin provide an increased protection against $S$. aureus infection (51).

\section{Antimicrobial Mechanisms of RNase 7 and Influence of the Ribonuclease Inhibitor}

As described above, RNase 7 is a member of the RNase A superfamily and a potent ribonuclease that is able to degrade
RNA. This raises the question of whether the ribonuclease activity is essential for the antimicrobial activity of RNase 7 . Huang et al. designed RNase 7 mutants (H15A, K38A, and H123A) lacking ribonuclease activity and showed that these mutants are still able to kill $P$. aeruginosa. In addition, they identified three clusters of cationic residues on the surface of RNase 7. Substitution experiments of cationic residues identified the first $\mathrm{N}$-terminal cluster with the lysine residues $\mathrm{K} 1, \mathrm{~K} 3$, K111, and K112 as essential for antimicrobial activity. The other two clusters located on rigid secondary structures did not influence antimicrobial activity of RNase 7 (37). In general, the $\mathrm{N}$-terminal cluster of RNases from the RNase A superfamily is the conserved region responsible for their antimicrobial activity (44). In line with the ribonuclease-independent killing activity against $P$. aeruginosa, we constructed a recombinant RNase 7 mutant without ribonuclease activity and showed that this mutant was still able to kill E. faecium (16). Ribonucleaseindependent antibacterial activity has also been described for RNase 3/ECP (52).

The ribonuclease activity of RNases from the RNase A superfamily can be inhibited by binding to the $50 \mathrm{kDa}$, cytosolic, horseshoe-shaped Ribonuclease Inhibitor (RI) in a 1:1 molar ratio. The RI forms tight complexes with the RNases which is known as one of the tightest formation in biological systems (53). It is an obvious question if binding of RI influences the antimicrobial activity of RNase 7. Addition of RI to RNase 7 suspensions and subsequent testing of the killing capacity of RNase 7 toward C. albicans and E. faecium resulted in a reduced antimicrobial activity indicating that the $\mathrm{RI}$ blocks the antimicrobial activity of RNase 7 (6). This inhibition may be due to steric interaction and not due to specific inhibition of the ribonuclease activity because the ribonuclease activity is not essential for antibacterial killing as described above. In concordance with these results, Spencer et al. also reported that the RI bound to RNase 7 and inhibited its antimicrobial activity by blocking its ability to bind the cell wall of uropathogenic bacteria (54).

Immunohistochemical analysis revealed that the RI is located mainly in the suprabasal epidermal layers but is absent in the stratum corneum. In addition, incubation of the RI with stratum corneum extracts led to the degradation of the RI suggesting that proteolytic activity of the stratum corneum degrades the RI (6). In contrast to the RI, RNase 7 is abundant in the stratum corneum. This led to the hypothesis that in the epidermis RNase 7 is complexed with the RI and thereby inactivated. Degradation of the RI in the stratum corneum hinders RImediated inactivation of RNase 7 thereby liberating RNase 7 to function as antimicrobial factor $(6,55)$.

The exact mechanisms underlying the antimicrobial activity of RNase 7 are not fully understood. Huang and coworkers performed binding experiments with DNA-binding SYTOX ${ }^{\circledR}$ Green dye and showed that RNase 7 is able to bind to the negatively charged membrane of $P$. aeruginosa and permeabilize the bacterial membrane (37). Torrent et al. demonstrated by means of liposome leakage assays that RNase 7 provokes membrane disruption by binding to bacterial membranes. Further experiments showed that RNase 7 did not interact with 
uncharged liposomes. Because of this fact the authors deduced that this membrane interaction is electrostatically driven (56). Further bacterial studies by this group showed a high leakage affinity of RNase 7 for $S$. aureus and E. coli membranes. RNase 7 depolarized the membrane by binding to lipopolysaccharide (LPS) and peptidoglycan, both major components of bacterial cell membranes from Gram-positive and Gram-negative bacteria, respectively (46). Another study from Lin et al. identified OprI (outer membrane protein I), an outer membrane lipoprotein from $P$. aeruginosa, as the initial binding site for RNase 7 instead of LPS. Addition of exogenous OprI or an anti-OprI antibody inhibited the antimicrobial activity of RNase 7 against $P$. aeruginosa. The authors conclude that upon RNase 7 binding, OprI internalizes along with RNase 7 into the cell leaving the cell membrane permeable to metabolites (49). These studies suggest that the interaction of RNase 7 with bacteria could be based on a target specific accumulation to different bacterial lipoproteins. This hypothesis needs to be proven in further studies with other bacteria.

In addition to its antibacterial activity, RNase 7 exhibits also activity against the yeast $C$. albicans. Salazar et al. described a dual mode of action for RNase 3 and RNase 7 as antifungal proteins against $C$. albicans. They designed mutants of both RNases by depleting the active catalytic site at His 15 which is the complement to His12 and performed depolarization and permeabilization membrane assays to assess the antimicrobial action against $C$. albicans. They could show that both RNases act first as membrane lytic proteins followed by enzymatic cellular RNA degradation (41).

\section{IMMUNOMODULATORY ACTIVITIES}

Besides its antimicrobial activity, some studies provide evidence for an additional immunomodulatory function of RNase 7 . Several members of the RNase A superfamily are well-known for their immunomodulatory activities (2). Although the role of RNase 7 as an immunomodulatory mediator is still emerging, there is first evidence of immunomodulatory functions associated with RNase 7. Resident and transiently migrating cells of the immune system are present in healthy human skin. With regard to antimicrobial defense, plasmacytoid dendritic cells (pDCs) are considered to play a key role within the types of the dermal immune system. pDCs usually circulate in the blood stream and are present in lymph nodes. Under inflammatory conditions they are able to infiltrate the skin (57). Due to their ability to secrete a several times stronger interferon-alpha (IFN $\alpha)$ response than any other cell types $(58,59)$, pDCs are crucial for cellular antimicrobial defense. A recent study from Kopfnagel et al. showed that RNase 7 in combination with human self-DNA activates a potent IFN $\alpha$ response in pDCs (60). This finding is in accordance with the known IFN $\alpha$-inducing ability of LL-37 (61) and hBD-2/-3 (62) in complex with self-DNA. In addition, comparative experiments revealed a markedly stronger IFN $\alpha$ production in response to RNase 7 mixed with self-DNA as compared to stimulation with LL-37 or hBD-2 in mixture with self-DNA (60). Of note, the amount of the RNase 7-induced
IFN $\alpha$ release was high enough to efficiently protect human keratinocytes from herpes simplex type I ( HSV-I) infection. This was of special significance because RNase 7 itself exhibits no direct antiviral activity against $H S V-I$. In summary, this study revealed the ability of RNase 7 to convert usually not immunogenic self-DNA into a danger signal which enables a strong immunomodulatory response (60). It remains to be shown if these characteristics of RNase 7 may be linked with autoinflammatory diseases.

$\mathrm{T}$ cells are present in human skin under healthy and inflammatory conditions. In healthy human skin they are of importance in wound healing, stimulate production of AMP by keratinocytes and influence keratinocyte development (63). Under inflammatory conditions $\mathrm{T}$ cells present in the blood can rapidly infiltrate the skin and produce proinflammatory cytokines, like IL-4 or interferon-gamma (64). A study of Kopfnagel et al. investigated the influence of RNase 7 on Th2 cytokine production by human CD4+T cells and Th2 cells. They reported that RNase 7 stimulation lead to a significantly reduced Th2 cytokine release of IL4, IL-5, and IL-13. This downregulation of Th2 cytokines was found to be mediated by a reduced activation of the transcription factor GATA3. Interestingly, the ribonuclease activity of RNase 7 was dispensable for this effect. Due to this specific regulation the authors assumed a yet unknown receptor-mediated process which needs to be elucidated in further studies (65).

A novel study documents that RNase 7 mediates recognition of self-DNA by human keratinocytes. Activation of keratinocytes by a DNA/RNase 7 complex resulted in a markedly increased release of the chemokine IP-10 (CXCL10), a process that was mediated by type I interferons. In addition, the stimulation of keratinocytes with RNase 7 and DNA induced an interferonbeta (IFNß) dependent antiviral response which was sufficient to counteract an infection of the keratinocytes with herpes-simplex virus 1 (HSV-1). Taken together, there is increasing evidence that RNase 7 is able to bind self-DNA immediately after its release and induces a rapid DNA-mediated activation of keratinocytes (66) and pDCs (60). Thus, RNase 7 may serve as an alarmin which detects a disruption of the skin barrier by converting released self-DNA into a danger signal (66).

Various immunomodulatory activities of other RNases of the human RNase A superfamily have been described. Examples are processing and clearance of RNA, activation of immune cells, chemotactic activities, angiogenesis and neovascularization, tissue remodeling and repair, wound healing activity, and induction of apoptosis [reviewed in (67)]. Thus, it is likely that RNase 7 exhibits additional, as yet unknown, immunomodulatory functions. The known immunomodulatory activities of RNase 7 are summarized in Table $2(60,65,66)$. Since the expression of RNase 7 is induced in inflammatory skin diseases such as psoriasis and atopic dermatitis (see below) it is likely that the immunomodulatory activities of RNase 7 may play a role in these skin diseases. Furthermore, it is also of interest to evaluate if the ribonuclease activity of RNase 7 may play a role in degrading host RNA released from damaged cells thereby controlling RNA-mediated inflammation during skin injury. 
TABLE 2 | Overview of the main findings regarding immunoregulatory activity of RNase 7.

\section{Findings}

References

Up-regulation of IFN $\alpha$ by human pDCs stimulated with

(60)

RNase 7 in mixture with self-DNA

Down-regulation of Th2 cytokines (IL-4, IL-13, and IL-5)

by activated human RNase 7-stimulated Th2 cells and

CD4+ T cells

RNase 7 stimulation of activated T cells results in a

reduced activity of the transcription factor GATA3

RNase 7 mediates sensing of self-DNA in human

keratinocytes leading to an antiviral immune response

\section{ROLE OF RNase 7 IN SKIN DISEASES}

\section{Atopic Dermatitis}

It has long been assumed that in the chronic inflammatory skin disease atopic dermatitis (AD) the expression of AMP including RNase 7 is impaired. This is based on the observation that a Th2 cytokine predominance has been shown to negatively regulate AMP expression $(68,69)$. In addition, comparative studies showed a reduced expression of AMP in AD skin as compared to psoriasis skin (70). However, especially for RNase 7 , growing evidence reveals an increased expression in AD skin as compared to healthy skin. By investigating skin biopsies using quantitative real-time PCR, Gambichler et al. showed a significantly higher expression of RNase 7 mRNA as compared to expression in healthy skin (71). In addition, Harder et al. investigated the protein expression of RNase 7 in skin biopsies using immunostaining and ELISA of skin-derived washing fluids. Both methods revealed an enhanced RNase 7 expression and secretion in lesional skin of $\mathrm{AD}$ patients as compared to healthy controls (23). In another study, Clausen et al. investigated RNase 7 protein expression in the uppermost skin layers using tape stripping followed by ELISA measurements. In line with the above mentioned studies they also found a higher expression of RNase 7 in AD skin as compared to healthy skin (72). Taken together, there is consistent evidence that expression of RNase 7 is induced in the skin of atopic dermatitis patients. The consequence of this induction on diseases progression remains to be analyzed in future studies.

As mentioned above, RNase 7 is able to downregulate Th2 cytokine production in CD4+ T cells. This effect was reduced in CD4+ T cells derived from AD patients (65). These data suggest that RNase 7 plays an important role to negatively regulate the expression of Th2 cytokines, a function that may be disturbed in AD thereby promoting a Th2 cytokine environment.

Filaggrin is an essential component of the skin barrier. A deficiency of this protein can lead to an impaired skin barrier function and this is assumed to be a major predisposing factor in the development of $\mathrm{AD}$ (73). van Drongelen et al. used 3D epidermal models with filaggrin knockdown and infected these with methicillin-resistant $S$. aureus (MRSA) bacteria. They showed that IL-31 favored epidermal S. aureus colonization by preventing the $S$. aureus-induced expression of RNase 7 and other AMP (26). IL-31 is a pruritus-causing cytokine with increased presence in AD (74). Thus, enhanced levels of IL-31 in $\mathrm{AD}$ may locally inhibit an adequate induction of RNase 7 in $S$. aureus-infected areas.

\section{Psoriasis}

A high-performance liquid chromatography (HPLC)-based analysis detected high amounts of RNase 7 and other AMP in psoriatic scale extracts (24). In line with these data, analyses of RNase 7 protein expression by immunohistochemistry and ELISA revealed increased expression of RNase 7 in the lesional skin of psoriasis patients (23). The increased amount of RNase 7 and other AMP in psoriasis may offer an explanation as to why psoriasis patients do not often suffer from skin infections despite the disturbed skin barrier (75).

One characteristic of psoriasis is the enrichment of pDCs in lesional skin and their key role in driving the development of the disease by the release of IFN $\alpha$ (76). As mentioned above, Kopfnagel et al. showed that RNase 7 in complex with self-DNA is a potent trigger of $\mathrm{pDC}$-derived IFN $\alpha$. Thus, an enhanced production of RNase 7 may trigger inflammation in psoriasis through increased production of IFN $\alpha$ (60). Similarly, the ability of RNase 7 to activate an inflammatory and antiviral response in keratinocytes in the presence of self-DNA may have implications in skin diseases such as psoriasis and atopic dermatitis where self-DNA derived from injured cells may be present in increased amounts (66).

\section{Dermatomycoses}

Major fungal cutaneous infections are superficial tinea and pityriasis versicolor which are caused by dermatophytes and Malassezia spp., respectively. An immunohistochemistry study from Brasch et al. investigated epidermal AMP expression in skin biopsies from infected and healthy persons. RNase 7 staining was significantly more often positive in the stratum granulosum of tinea than in the stratum granulosum of pityriasis versicolor and normal skin (77). In concordance with these findings, Firat et al. detected a high upregulation of RNase 7 expression in keratinocytes infected with T. rubrum (30). By blocking the EGFR, the T. rubrum-mediated RNase 7 induction in keratinocytes was significantly reduced. Interestingly, patients with anti-EGFR therapy have a high prevalence of cutaneous infections including those caused by dermatophytes such as $T$. rubrum (78). One may speculate that the anti-EGFR therapy may impair antifungal defense of the skin by inhibiting production of AMP such as RNase 7.

\section{S. aureus Skin Infections}

As already described above, RNase 7 exhibits potent in vitro anti-staphylococcal activity and ex vivo as well as in vivo studies highlight an important role of RNase 7 to control the growth of $S$. aureus. This gives rise to the speculation that RNase 7 may make up a fundamental part of innate cutaneous defense to fight off $S$. aureus infections and that a dysregulation of RNase 7 in general enhances the susceptibility toward S. aureus infections. However, this intriguing hypothesis has to be verified in further studies. 
Chronic, recurrent, and persistent infections caused by $S$. aureus are often associated with the formation of the smallcolony variant (SCV) phenotype. SCV have a longer generation time leading to small colonies on agar plates. It is believed that a switch from the wild-type phenotype to a SCV phenotype makes it possible for $S$. aureus to escape from host defense and to spread the infection (79). To proof the hypothesis that SCV are less susceptible to the cutaneous AMP-mediated killing activity, Gläser et al. used clinical S. aureus SCV strains and exposed them to either AMP directly or to stratum corneum extracts from healthy donors. In both setups the killing activity toward S. aureus isolates displaying the SCV phenotype was markedly reduced (43). These experiments demonstrated a less susceptibility of SCV to the antimicrobial activity of RNase 7 and other human skin-derived AMP. Thus, switching into the SCV phenotype may help $S$. aureus to subvert cutaneous innate defense through a higher resistance toward AMP such as RNase 7.

An increasing threat is the spread of MRSA. MRSA acquired resistance mechanisms against many antibiotics commonly used to treat $S$. aureus skin infections. In human skin equivalents infected with MRSA the expression of RNase 7 was induced, in particular in wounded skin (28). Of note, RNase 7 is able to dampen the growth of MRSA in vitro (12) and own unpublished results. These data indicate an important role of RNase 7 as cutaneous defense factor to control the growth of MRSA. It is an interesting hypothesis that patients with an impaired RNase 7-based cutaneous defense are more susceptible to the spread of virulent $S$. aureus including MRSA. Notably, the highly

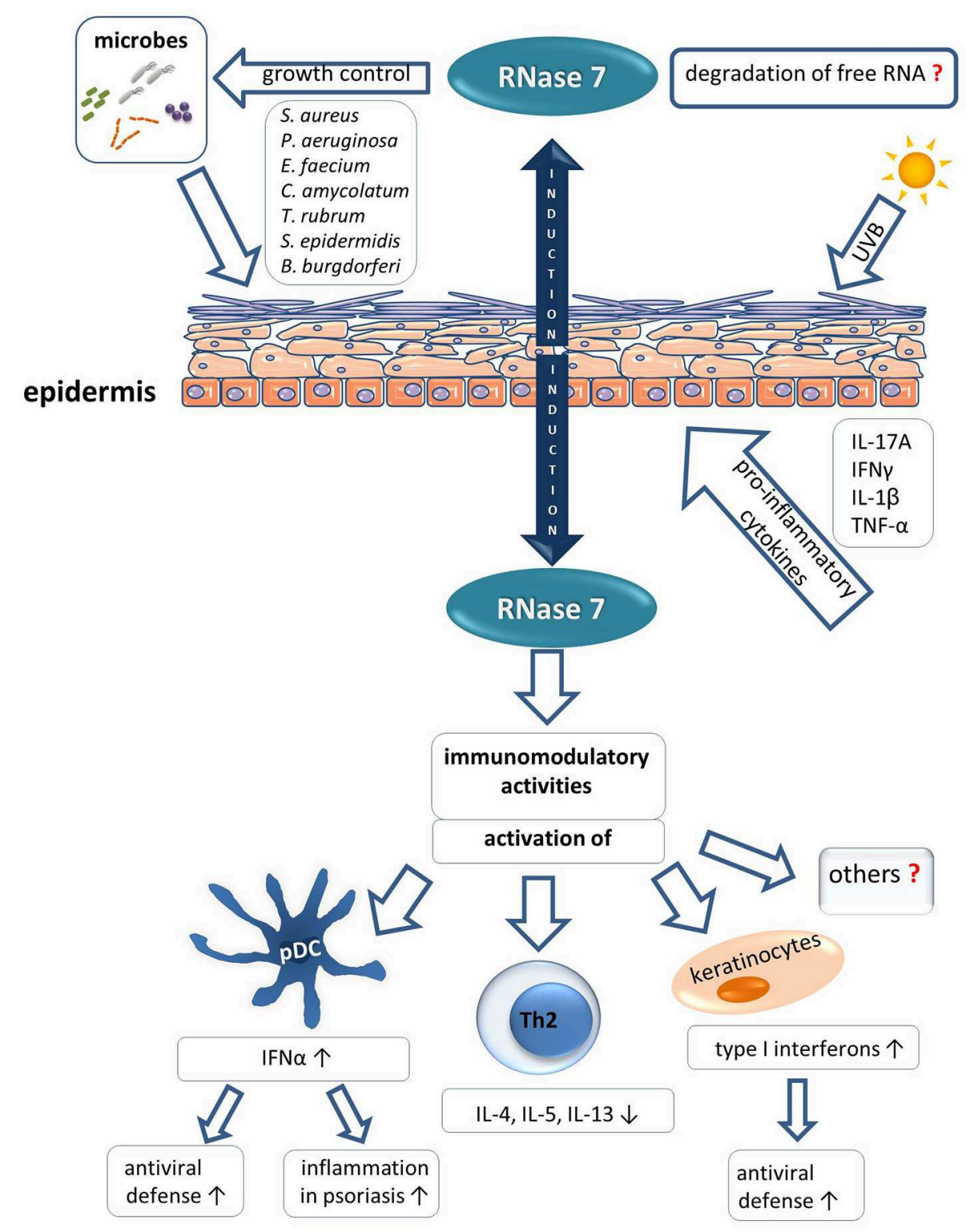

FIGURE 2 | Role of RNase 7 in skin defense. 
virulent strain USA300, a highly pathogenic MRSA causing severe skin and soft tissue infections, displays a comparatively low susceptibility toward the anti-staphylococcal activity of stratum corneum extracts (42). If a decreased activity of RNase 7 against USA300 contributes to this effect is speculative but not unlikely. This is supported by the fact that RNase 7 is a major component of the anti-staphylococcal activity of stratum corneum skin extracts (25).

As discussed above the presence of cationic amino acid residues endows RNase 7 with a positively charged N-terminal cluster that is required for antimicrobial activity. It is known that $S$. aureus has the capability to reduce its susceptibility toward the action of cationic AMP through the reduction of its negative surface charge. This can be achieved by the incorporation of $\mathrm{D}$-alanine in its teichoic acids, a mechanism that lowered the susceptibility of $S$. aureus toward several skin-derived AMP including RNase 7 (42). It remains to be determined if the use of such strategies to lower the sensitivity toward the bactericidal activity of RNase 7 are associated with a higher pathogenic potential of distinct $S$. aureus strains.

\section{Wounds}

An intact skin barrier is essential for the protection against potential pathogenic microorganisms. In case of wounding, a rapid and potent defense is pivotal. In this regard, it has been shown that RNase 7 is rapidly released on the skin surface after experimental superficial barrier disruption (23). In contrast, Dressel et al. detected no increase of RNase 7 expression in the margins of chronic wounds, whereas the expression of the AMP hBD-2 and psoriasin was strongly induced. This led to the hypothesis that an insufficient expression of RNase 7 in chronic wounds may contribute to disturbed wound healing (80). Zanger et al. reported on a 50\% lower RNase 7 gene expression in skin 3 days after sterile wounding (81). This in turn supports the idea that RNase 7 acts primarily as a component of the early and primary cutaneous defense response after injury (81). Clearly, more studies are needed to decipher the antimicrobial and immunomodulatory functions of RNase 7 in wounded skin.

\section{Mycobacterial Infections}

It has been reported that RNase 7 exhibits antimicrobial activity against Mycobacterium vaccae at low micromolar concentrations (40). Furthermore, infection of airway epithelial cells with Mycobacterium tuberculosis led to induction of RNase 7 expression and an intracellular association of RNase 7 with Mycobacterium tuberculosis (50). This may suggest a direct antimicrobial effect of RNase 7 on Mycobacterium tuberculosis, but this has to be confirmed in further studies. Nevertheless, these initial studies give rise to the hypothesis that RNase 7 may play a role in infections caused by mycobacteria. Thus, it remains to be shown whether RNase 7 may also be involved in cutaneous mycobacterial defense (82).

\section{OUTLOOK}

The role of RNase 7 in the skin is still emerging. However, as outlined in this review there is increasing evidence that
RNase 7 plays an important role in innate cutaneous defense. This is mediated by the antimicrobial and immunomodulatory characteristics of RNase 7 (Figure 2 summarizes the role of RNase 7 in skin defense). It is likely that future studies will reveal novel immunomodulatory functions of RNase 7 and shed light on the as yet poorly understood importance of the enzymatic activity of RNase 7 in a physiological context. Moreover, it is of importance to further elucidate the potential link between specific infectious and inflammatory diseases and an impaired expression and/or function of RNase 7.

As mentioned in this review, functional studies as well as in vivo observations identified RNase 7 as a crucial factor to control the growth of the major cutaneous pathogen $S$. aureus. There is also evidence that other pathogens such as $P$. aeruginosa are affected by RNase 7 . Thus, it is an interesting hypothesis that RNase 7 or optimized derivatives based on RNase 7 may have a high potential as anti-invectives. In this regard, the in vitro activity of RNase 7 against multiresistant bacteria (12) may offer a promising alternative to fight off bacteria that are hard to kill by common antibiotics. Moreover, the targeted design of chimeric constructs combining selected parts of different molecules with high antimicrobial and ribonuclease activity, as recently reported for an RNase $3 / 1$ hybrid construct (83), is a promising strategy. However, when considering RNase 7 for application as a therapeutic drug it is of great importance to evaluate the possibility that an excessive medical use of RNase 7 may induce the emergence of bacteria with an acquired resistance toward RNase 7. Such scenario could threaten our own innate host defense. In addition, the influence of immunomodulatory activities as well as the interaction of RNase 7 with the microbiota have to be considered when using RNase 7 in the treatment or prophylaxis of infections. In the case of atopic dermatitis, where $S$. aureus and elevated Th2 cytokines are major drivers of the disease, the capability of RNase 7 to kill $S$. aureus together with its influence on $\mathrm{T}$ cells to dampen secretion of Th2 cytokines may qualify RNase 7 as a beneficial drug to treat atopic dermatitis. Finally, the inducibility of RNase 7 gives rise to the speculation that a targeted induction of RNase 7 may offer a useful treatment or prophylactic option. Clearly, more studies are needed to unravel the physiological role of RNase 7 and to define its role in diseases and as a potential drug.

\section{AUTHOR CONTRIBUTIONS}

FR, SD, and JH wrote the original draft and edited versions. VK, $\mathrm{RG}$, and TW edited versions. FR and JH prepared the figures.

\section{FUNDING}

Research work was supported by grants of the German research foundation (DFG) HA 3386/8-1 and WE1289/10-1. The work of TW has been funded by the German research foundation (DFG) under Germany's Excellence Strategy-EXC 2155 RESISTProject ID 39087428. 


\section{REFERENCES}

1. Sorrentino S. The eight human "canonical" ribonucleases: molecular diversity, catalytic properties, and special biological actions of the enzyme proteins. FEBS Lett. (2010) 584:2194-200. doi: 10.1016/j.febslet.2010.04.018

2. Schwartz L, Cohen A, Thomas J, Spencer JD. The immunomodulatory and antimicrobial properties of the vertebrate ribonuclease A superfamily. Vaccines. (2018) 6:76. doi: 10.3390/vaccines6040076

3. Malik A, Batra JK. Antimicrobial activity of human eosinophil granule proteins: involvement in host defence against pathogens. Crit Rev Microbiol. (2012) 38:168-81. doi: 10.3109/1040841X.2011.645519

4. Koczera P, Martin L, Marx G, Schuerholz T. The ribonuclease A superfamily in humans: canonical RNases as the buttress of innate immunity. Int J Mol Sci. (2016) 17:1278. doi: 10.3390/ijms 17081278

5. Hooper LV, Stappenbeck TS, Hong CV, Gordon JI. Angiogenins: a new class of microbicidal proteins involved in innate immunity. Nat Immunol. (2003) 4:269-73. doi: 10.1038/ni888

6. Abtin A, Eckhart L, Mildner M, Ghannadan M, Harder J, Schröder J-M, et al. Degradation by stratum corneum proteases prevents endogenous RNase inhibitor from blocking antimicrobial activities of RNase 5 and RNase 7. J Invest Dermatol. (2009) 129:2193-201. doi: 10.1038/jid.2009.35

7. Becknell B, Eichler TE, Beceiro S, Li B, Easterling RS, Carpenter AR, et al. Ribonucleases 6 and 7 have antimicrobial function in the human and murine urinary tract. Kidney Int. (2015) 87:151-61. doi: 10.1038/ki.2014.268

8. Rademacher F, Simanski M, Harder J. RNase 7 in cutaneous defense. Int J Mol Sci. (2016) 17:560. doi: 10.3390/ijms17040560

9. Becknell B, Spencer JD. A review of ribonuclease 7's structure, regulation, and contributions to host defense. Int J Mol Sci. (2016) 17:423. doi: 10.3390/ijms17030423

10. Rudolph B, Podschun R, Sahly H, Schubert S, Schröder JM, Harder J. Identification of RNase 8 as a novel human antimicrobial protein. Antimicrob Agents Chemother. (2006) 50:3194-6. doi: 10.1128/AAC.00246-06

11. Simanski M, Köten $B$, Schröder J-M, Gläser R, Harder J. Antimicrobial RNases in cutaneous defense. J Innate Immun. (2012) 4:241-7. doi: 10.1159/000335029

12. Harder J, Schröder J-M. RNase 7, a novel innate immune defense antimicrobial protein of healthy human skin. J Biol Chem. (2002) 277:4677984. doi: 10.1074/jbc.M207587200

13. Zhang J, Dyer KD, Rosenberg HF. Human RNase 7: a new cationic ribonuclease of the RNase A superfamily. Nucleic Acids Res. (2003) 31:6027. doi: $10.1093 /$ nar/gkg157

14. Spencer JD, Schwaderer AL, Dirosario JD, McHugh KM, McGillivary G, Justice SS, et al. Ribonuclease 7 is a potent antimicrobial peptide within the human urinary tract. Kidney Int. (2011) 80:174-80. doi: 10.1038/ki.2011.109

15. Eichler T, Bender K, Murtha MJ, Schwartz L, Metheny J, Solden L, et al. Ribonuclease 7 shields the kidney and bladder from invasive uropathogenic Escherichia coli infection. J Am Soc Nephrol. (2019) 30:138597. doi: 10.1681/ASN.2018090929

16. Köten B, Simanski M, Gläser R, Podschun R, Schröder J-M, Harder J. RNase 7 contributes to the cutaneous defense against Enterococcus faecium. PLoS ONE. (2009) 4:e6424. doi: 10.1371/journal.pone.00 06424

17. Reithmayer K, Meyer KC, Kleditzsch P, Tiede S, Uppalapati SK, Gläser R, et al. Human hair follicle epithelium has an antimicrobial defence system that includes the inducible antimicrobial peptide psoriasin (S100A7) and RNase 7. Br J Dermatol. (2009) 161:78-89. doi: 10.1111/j.1365-2133.2009. 09154.x

18. Wittersheim M, Cordes J, Meyer-Hoffert U, Harder J, Hedderich J, Gläser R. Differential expression and in vivo secretion of the antimicrobial peptides psoriasin (S100A7), RNase 7, human beta-defensin-2 and-3 in healthy human skin. Exp Dermatol. (2013) 22:364-6. doi: 10.1111/exd.12133

19. Schuster C, Gläser R, Fiala C, Eppel W, Harder J, Schröder J-M, et al. Prenatal human skin expresses the antimicrobial peptide RNase 7. Arch Dermatol Res. (2013) 305:545-9. doi: 10.1007/s00403-013-1340-y

20. Simanski M, Rademacher F, Schröder L, Schumacher H, Gläser R, Harder J. IL-17A and IFN- $\gamma$ synergistically induce RNase 7 expression via STAT3 in primary keratinocytes. PLoS ONE. (2013) 8:e59531. doi: 10.1371/journal.pone.0059531
21. Mohammed I, Yeung A, Abedin A, Hopkinson A, Dua HS. Signalling pathways involved in ribonuclease-7 expression. Cell Mol Life Sci. (2011) 68:1941-52. doi: 10.1007/s00018-010-0540-2

22. Burgey C, Kern WV, Römer W, Rieg S. Differential induction of innate defense antimicrobial peptides in primary nasal epithelial cells upon stimulation with inflammatory cytokines, Th17 cytokines or bacterial conditioned medium from Staphylococcus aureus isolates. Microb Pathog. (2016) 90:6977. doi: 10.1016/j.micpath.2015.11.023

23. Harder, Dressel S, Wittersheim M, Cordes J, Meyer-Hoffert U, Mrowietz $\mathrm{U}$, et al. Enhanced expression and secretion of antimicrobial peptides in atopic dermatitis and after superficial skin injury. J Invest Dermatol. (2010) 130:1355-64. doi: 10.1038/jid.2009.432

24. Harder J, Schröder JM. Psoriatic scales: a promising source for the isolation of human skin-derived antimicrobial proteins. J Leukoc Biol. (2005) 77:47686. doi: 10.1189/jlb.0704409

25. Simanski, Dressel S, Gläser R, Harder J. RNase 7 protects healthy skin from Staphylococcus aureus colonization. J Invest Dermatol. (2010) 130:28368. doi: 10.1038/jid.2010.217

26. van Drongelen V, Haisma EM, Out-Luiting JJ, Nibbering PH, El Ghalbzouri A. Reduced filaggrin expression is accompanied by increased Staphylococcus aureus colonization of epidermal skin models. Clin Exp Allergy. (2014) 44:1515-24. doi: 10.1111/cea.12443

27. Wanke I, Steffen H, Christ C, Krismer B, Götz F, Peschel A, et al. Skin commensals amplify the innate immune response to pathogens by activation of distinct signaling pathways. J Invest Dermatol. (2011) 131:38290. doi: 10.1038/jid.2010.328

28. Haisma EM, Rietveld MH, de Breij A, van Dissel JT, El Ghalbzouri A, Nibbering PH. Inflammatory and antimicrobial responses to methicillinresistant Staphylococcus aureus in an in vitro wound infection model. PLoS ONE. (2013) 8:e82800. doi: 10.1371/journal.pone.0082800

29. Rademacher F, Simanski M, Schröder L, Mildner M, Harder J. The role of RNase 7 in innate cutaneous defense against Pseudomonas aeruginosa. Exp Dermatol. (2017) 26:227-33. doi: 10.1111/exd.13166

30. Firat YH, Simanski M, Rademacher F, Schröder L, Brasch J, Harder J. Infection of keratinocytes with Trichophytum rubrum induces epidermal growth factordependent RNase 7 and human beta-defensin-3 expression. PLoS ONE. (2014) 9:e93941. doi: 10.1371/journal.pone.0093941

31. Walter S, Rademacher F, Kobinger N, Simanski M, Gläser R, Harder J. RNase 7 participates in cutaneous innate control of Corynebacterium amycolatum. Sci Rep. (2017) 7:13862. doi: 10.1038/s41598-017-14383-Z

32. Lichtenberger BM, Gerber PA, Holcmann M, Buhren BA, Amberg $\mathrm{N}$, Smolle V, et al. Epidermal EGFR controls cutaneous host defense and prevents inflammation. Sci Transl Med. (2013) 5:199ral11. doi: 10.1126/scitranslmed.3005886

33. Marchal C, Schramm F, Kern A, Luft BJ, Yang X, Schuijt T, et al. Antialarmin effect of tick saliva during the transmission of lyme disease. Infect Immun. (2011) 79:774-85. doi: 10.1128/IAI.00482-10

34. Surasombatpattana P, Hamel R, Patramool S, Luplertlop N, Thomas F, Desprès $\mathrm{P}$, et al. Dengue virus replication in infected human keratinocytes leads to activation of antiviral innate immune responses. Infect Genet Evol. (2011) 11:1664-73. doi: 10.1016/j.meegid.2011.06.009

35. Gläser R, Navid F, Schuller W, Jantschitsch C, Harder J, Schröder JM, et al. UV-B radiation induces the expression of antimicrobial peptides in human keratinocytes in vitro and in vivo. J Allergy Clin Immunol. (2009) 123:111723. doi: 10.1016/j.jaci.2009.01.043

36. Mathapathi MS, Mallemalla P, Vora S, Iyer V, Tiwari JK, Chakrabortty A, et al. Niacinamide leave-on formulation provides long-lasting protection against bacteria in vivo. Exp Dermatol. (2017) 26:827-9. doi: 10.1111/exd.13285

37. Huang Y-C, Lin Y-M, Chang T-W, Wu S-J, Lee Y-S, Chang MD-T, et al. The flexible and clustered lysine residues of human ribonuclease 7 are critical for membrane permeability and antimicrobial activity. J Biol Chem. (2007) 282:4626-33. doi: 10.1074/jbc.M607321200

38. Cho JS, Xuan C, Miller LS. Lucky number seven: RNase 7 can prevent Staphylococcus aureus skin colonization. J Invest Dermatol. (2010) 130:27036. doi: 10.1038/jid.2010.294

39. Fritz P, Beck-Jendroschek V, Brasch J. Inhibition of dermatophytes by the antimicrobial peptides human $\beta$-defensin-2, ribonuclease 7 and psoriasin. Med Mycol. (2012) 50:579-84. doi: 10.3109/13693786.2012.660203 
40. Pulido D, Torrent M, Andreu D, Nogues MV, Boix E. Two human host defense ribonucleases against mycobacteria, the eosinophil cationic protein (RNase 3) and RNase 7. Antimicrob Agents Chemother. (2013) 57:3797805. doi: 10.1128/AAC.00428-13

41. Salazar VA, Arranz-Trullén J, Navarro S, Blanco JA, Sánchez D, Moussaoui $\mathrm{M}$, et al. Exploring the mechanisms of action of human secretory RNase 3 and RNase 7 against Candida albicans. Microbiologyopen. (2016) 5:83045. doi: 10.1002/mbo3.373

42. Simanski M, Gläser R, Köten B, Meyer-Hoffert U, Wanner S, Weidenmaier C, et al. Staphylococcus aureus subverts cutaneous defense by D-alanylation of teichoic acids. Exp Dermatol. (2013) 22:294-6. doi: 10.1111/exd.12114

43. Gläser $\mathrm{R}$, Becker $\mathrm{K}$, von Eiff $\mathrm{C}$, Meyer-Hoffert U, Harder J. Decreased susceptibility of Staphylococcus aureus small-colony variants toward human antimicrobial peptides. J Invest Dermatol. (2014) 134:2347-50. doi: 10.1038/jid.2014.176

44. Torrent M, Pulido D, Valle J, Nogués MV, Andreu D, Boix E. Ribonucleases as a host-defence family: evidence of evolutionarily conserved antimicrobial activity at the N-terminus. Biochem J. (2013) 456:99-108. doi: 10.1042/BJ20130123

45. Köten B, Becker K, Podschun R, Von Eiff C, Meyer-Hoffert U, Harder J, et al. Susceptibility of Staphylococcus aureus bacteremia strains to different skin-derived antimicrobial proteins. Arch Dermatol Res. (2012) 304:6337. doi: 10.1007/s00403-012-1284-7

46. Torrent M, Badia M, Moussaoui M, Sanchez D, Nogués MV, Boix E. Comparison of human RNase 3 and RNase 7 bactericidal action at the Gramnegative and Gram-positive bacterial cell wall. FEBS J. (2010) 277:171325. doi: 10.1111/j.1742-4658.2010.07595.x

47. Wang H, Schwaderer AL, Kline J, Spencer JD, Kline D, Hainsa DS. Contribution of structural domains to the activity of ribonuclease 7 against uropathogenic bacteria. Antimicrob Agents Chemother. (2013) 57:76674. doi: 10.1128/AAC.01378-12

48. Spencer JD, Schwaderer AL, Wang H, Bartz J, Kline J, Eichler T, et al. Ribonuclease 7, an antimicrobial peptide upregulated during infection, contributes to microbial defense of the human urinary tract. Kidney Int. (2013) 83:615-25. doi: 10.1038/ki.2012.410

49. Lin Y-M, Wu S-J, Chang T-W, Wang C-F, Suen C-S, Hwang $\mathrm{M}-\mathrm{J}$, et al. Outer membrane protein I of Pseudomonas aeruginosa is a target of cationic antimicrobial peptide/protein. J Biol Chem. (2010) 285:8985-94. doi: 10.1074/jbc.M109.0 78725

50. Torres-Juarez F, Touqui L, Leon-Contreras J, Rivas-Santiago C, EncisoMoreno JA, Hernández-Pando R, et al. RNase 7 but not psoriasin nor sPLA2IIA associates with Mycobacterium tuberculosis during airway epithelial cell infection. Pathog Dis. (2018) 76:1-8. doi: 10.1093/femspd/fty005

51. Zanger P, Holzer J, Schleucher R, Steffen H, Schittek B, Gabrysch S. Constitutive expression of the antimicrobial peptide RNase 7 is associated with Staphylococcus aureus infection of the skin. J Infect Dis. (2009) 200:190715. doi: $10.1086 / 648408$

52. Rosenberg HF. Recombinant human eosinophil cationic protein. Ribonuclease activity is not essential for cytotoxicity. J Biol Chem. (1995) 270:7876-81. doi: 10.1074/jbc.270.14.7876

53. Dickson KA, Haigis MC, Raines RT. Ribonuclease inhibitor: structure and function. Prog Nucleic Acid Res Mol Biol. (2005) 80:349-74. doi: 10.1016/S0079-6603(05)80009-1

54. Spencer JD, Schwaderer AL, Eichler T, Wang H, Kline J, Justice SS, et al. An endogenous ribonuclease inhibitor regulates the antimicrobial activity of ribonuclease 7 in the human urinary tract. Kidney Int. (2014) 85:117991. doi: 10.1038/ki.2013.395

55. Zasloff M. Antimicrobial RNases of human skin. J Invest Dermatol. (2009) 129:2091-3. doi: 10.1038/jid.2009.216

56. Torrent M, Sánchez D, Buzón V, Nogués MV, Cladera J, Boix E. Comparison of the membrane interaction mechanism of two antimicrobial RNases: RNase 3/ECP and RNase 7. Biochim Biophys Acta. (2009) 1788:111625. doi: 10.1016/j.bbamem.2009.01.013

57. Donaghy H, Bosnjak L, Harman AN, Marsden V, Tyring SK, Meng T$\mathrm{C}$, et al. Role for plasmacytoid dendritic cells in the immune control of recurrent human herpes simplex virus infection. J Virol. (2009) 83:195261. doi: 10.1128/JVI.01578-08
58. Gilliet M, Cao W, Liu Y-J. Plasmacytoid dendritic cells: sensing nucleic acids in viral infection and autoimmune diseases. Nat Rev Immunol. (2008) 8:594-606. doi: 10.1038/nri2358

59. Siegal FP, Kadowaki N, Shodell M, Fitzgerald-Bocarsly PA, Shah K, Ho S, et al. The nature of the principal type 1 interferon-producing cells in human blood. Science. (1999) 284:1835-7. doi: 10.1126/science.284.5421.1835

60. Kopfnagel V, Wagenknecht S, Harder J, Hofmann K, Kleine M, Buch A, et al. RNase 7 strongly promotes TLR9-mediated DNA sensing by human plasmacytoid dendritic cells. J Invest Dermatol. (2018) 138:87281. doi: 10.1016/j.jid.2017.09.052

61. Lande R, Gregorio J, Facchinetti V, Chatterjee B, Wang Y-H, Homey B, et al. Plasmacytoid dendritic cells sense self-DNA coupled with antimicrobial peptide. Nature. (2007) 449:564-9. doi: 10.1038/nature06116

62. Tewary P, de la Rosa G, Sharma N, Rodriguez LG, Tarasov SG, Howard OM, et al. $\beta$-Defensin 2 and 3 promote the uptake of self or CpG DNA, enhance IFN- $\alpha$ production by human plasmacytoid dendritic cells, and promote inflammation. J Immunol. (2013) 191:86574. doi: 10.4049/jimmunol.1201648

63. Suwanpradid J, Holcomb ZE, MacLeod AS. Emerging skin T-cell functions in response to environmental insults. J Invest Dermatol. (2017) 137:28894. doi: 10.1016/j.jid.2016.08.013

64. Nguyen AV, Soulika AM. The dynamics of the skin's immune system. Int J Mol Sci. (2019) 20:1811. doi: 10.3390/ijms20081811

65. Kopfnagel V, Wagenknecht S, Brand L, Zeitvogel J, Harder J, Hofmann K, et al. RNase 7 downregulates TH2 cytokine production by activated human T cells. Allergy. (2017) 72:1694-703. doi: 10.1111/all.13173

66. Kopfnagel V, Wagenknecht S, Baumert K, Stark M, Harder J, Hofmann K, et al. RNase 7 promotes sensing of self-DNA by human keratinocytes and activates an antiviral immune response. J Invest Dermatol. (2019). [Epub ahead of print].

67. $\mathrm{Lu} \mathrm{L}$, Li J, Moussaoui M, Boix E. Immune modulation by human secreted RNases at the extracellular space. Front Immunol. (2018) 9:1012. doi: 10.3389/fimmu.2018.01012

68. Nomura I, Goleva E, Howell MD, Hamid QA, Ong PY, Hall CF, et al. Cytokine milieu of atopic dermatitis, as compared to psoriasis, skin prevents induction of innate immune response genes. J Immunol. (2003) 171:3262 9. doi: 10.4049/jimmunol.171.6.3262

69. Howell MD, Gallo RL, Boguniewicz M, Jones JF, Wong C, Streib $\mathrm{JE}$, et al. Cytokine milieu of atopic dermatitis skin subverts the innate immune response to vaccinia virus. Immunity. (2006) 24:341-8. doi: 10.1016/j.immuni.2006.02.006

70. Ong PY, Ohtake T, Brandt C, Strickland I, Boguniewicz M, Ganz T, et al. Endogenous antimicrobial peptides and skin infections in atopic dermatitis. N Engl J Med. (2002) 347:1151-60. doi: 10.1056/NEJMoa021481

71. Gambichler T, Skrygan M, Tomi NS, Othlinghaus N, Brockmeyer NH, Altmeyer P, et al. Differential mRNA expression of antimicrobial peptides and proteins in atopic dermatitis as compared to psoriasis vulgaris and healthy skin. Int Arch Allergy Appl Immunol. (2008) 147:1724. doi: $10.1159 / 000128582$

72. Clausen M-L, Slotved H-C, Krogfelt KA, Agner T. Measurements of AMPs in stratum corneum of atopic dermatitis and healthy skin-tape stripping technique. Sci Rep. (2018) 8:1666. doi: 10.1038/s41598-018-20204-8

73. Palmer CNA, Irvine AD, Terron-Kwiatkowski A, Zhao Y, Liao H, Lee SP, et al. Common loss-of-function variants of the epidermal barrier protein filaggrin are a major predisposing factor for atopic dermatitis. Nat Genet. (2006) 38:441-6. doi: 10.1038/ng1767

74. Furue M, Yamamura K, Kido-Nakahara M, Nakahara T, Fukui Y. Emerging role of interleukin-31 and interleukin-31 receptor in pruritus in atopic dermatitis. Allergy. (2018) 73:29-36. doi: 10.1111/all.13239

75. Henseler T, Christophers E. Disease concomitance in psoriasis. J Am Acad Dermatol. (1995) 32:982-6. doi: 10.1016/0190-9622(95)91336-X

76. Nestle FO, Conrad C, Tun-Kyi A, Homey B, Gombert M, Boyman O, et al. Plasmacytoid predendritic cells initiate psoriasis through interferonalpha production. J Exp Med. (2005) 202:135-43. doi: 10.1084/jem.200 50500

77. Brasch J, Mörig A, Neumann B, Proksch E. Expression of antimicrobial peptides and toll-like receptors is increased in tinea and pityriasis versicolor. Mycoses. (2014) 57:147-52. doi: 10.1111/myc.12118 
78. Eilers RE, Gandhi M, Patel JD, Mulcahy MF, Agulnik M, Hensing T, et al. Dermatologic infections in cancer patients treated with epidermal growth factor receptor inhibitor therapy. J Natl Cancer Inst. (2010) 102:4753. doi: 10.1093/jnci/djp439

79. Tuchscherr L, Medina E, Hussain M, Völker W, Heitmann V, Niemann S, et al. Staphylococcus aureus phenotype switching: an effective bacterial strategy to escape host immune response and establish a chronic infection. EMBO Mol Med. (2011) 3:129-41. doi: 10.1002/emmm.2010 00115

80. Dressel S, Harder J, Cordes J, Wittersheim M, Meyer-Hoffert U, Sunderkötter C, et al. Differential expression of antimicrobial peptides in margins of chronic wounds. Exp Dermatol. (2010) 19:628-32. doi: 10.1111/j.1600-0625.2009. 01030.x

81. Zanger P, Nurjadi D, Vath B, Kremsner PG. Persistent nasal carriage of Staphylococcus aureus is associated with deficient induction of human beta-defensin 3 after sterile wounding of healthy skin in vivo. Infect Immun. (2011) 79:2658-62. doi: 10.1128/IAI.00 101-11
82. Franco-Paredes C, Marcos LA, Henao-Martínez AF, Rodríguez-Morales AJ, Villamil-Gómez WE, Gotuzzo E, et al. Cutaneous mycobacterial infections. Clin Microbiol Rev. (2019) 32:1-25. doi: 10.1128/CMR.00069-18

83. Prats-Ejarque G, Li J, Ait-Ichou F, Lorente H, Boix E. Testing a human antimicrobial RNase chimera against bacterial resistance. Front Microbiol. (2019) 10:1357. doi: 10.3389/fmicb.2019.01357

Conflict of Interest: The authors declare that the research was conducted in the absence of any commercial or financial relationships that could be construed as a potential conflict of interest.

Copyright (c) 2019 Rademacher, Dreyer, Kopfnagel, Gläser, Werfel and Harder. This is an open-access article distributed under the terms of the Creative Commons Attribution License (CC BY). The use, distribution or reproduction in other forums is permitted, provided the original author(s) and the copyright owner(s) are credited and that the original publication in this journal is cited, in accordance with accepted academic practice. No use, distribution or reproduction is permitted which does not comply with these terms. 